\title{
FORMULAE FOR SOLID-ON-SOLID NUCLEATION MODEL
}

\author{
M. SUFFCZYŃSKI \\ Institute of Physics, Polish Academy of Sciences \\ Al. Lotników 32/46, Warszawa 02-668, Poland
}

(Received May 9, 1991)

\begin{abstract}
The solid-on-solid model, with nearest- and next-nearest neighbour interactions, of two-dimensional nucleation on the surface of a crystal in unstable equilibrium with its supersaturated vapour allows from the local mean direction of curved step to derive closed formulae for the shape, the area, and the activation energy for growth of the crystal nucleus. The formulae facilitate to estimate from the observed shape patterns the parameters of nucleation, to follow the evolution of the crystal nucleus with temperature and the dependence of activation energy on distance between screw dislocations which provide steps on the crystal surface.
\end{abstract}

PACS numbers: $61.50 . \mathrm{Cj}, 64.70 . \mathrm{Hz}$.

\section{Introduction}

Crystal nucleation and equilibrium crystal shapes in $D=2$ dimensions, with nearest-neighbour (NN) interaction between the atomic constituents, are described by the Ising model [1-5]. When the crystal lattice has square symmetry the Ising model free energy has proper fourfold symmetry.

To study equilibrium crystal shapes with the nearest- and next-nearest neighbour (NNN) interactions the solid-on-solid (SOS) approximation of Burton, Cabrera and Frank (BCF) [6] is used. The SOS model fails to possess the exact four-fold symmetry of the square lattice $[1-3,7]$ but the deviation from this symmetry is proportional to $T \exp \left(-J / k_{\mathrm{B}} T\right)$, thus is negligibly small at low temperature $T$ for typical values of the NN interaction energy parameter $J=\phi_{1} / 4$. In the SOS model of BCF the equilibrium crystal shape, the crystal nucleus area in $D=2$ dimensions and the activation energy for nucleation can be expressed by closed formulae which are derived below. 


\section{Equilibrium structure of step}

When the crystal bulk correlation length is small, the crystal interface can be modelled as a two-dimensional surface and the dominant fluctuations of this surface are steps or ledges [8-14]. A crystal in unstable equilibrium with its supersaturated vapour has curved steps appearing on the surface. BCF considered the surface of a simple cubic crystal with NN and NNN interaction energies $\phi_{1}$ and $\phi_{2}$, respectively. With the Boltzmann constant $k_{\mathrm{B}}$ and $\beta^{-1}=k_{\mathrm{B}} T$ the Boltzmann factors are $\eta_{1,2}=\exp \left(-\phi_{1,2} \beta / 2\right)$ in the notation of BCF. We write $\eta=\eta_{2}^{2}$. For a vapour saturation ratio $\alpha$ BCF give an explicit expression for the local mean direction of a curved step at point $x$. The angle $\theta(x)$ between the step and the $[0,1]$ crystal direction is given by

$$
\operatorname{tg} \theta(x)=\left(u-u^{-1}\right)\left[\left(u+u^{-1}-c_{2}\right)^{-1}-\left(u+u^{-1}+c_{5}\right)^{-1}\right],
$$

where $u=\alpha^{x}=\exp (x \ln \alpha)$, and

$$
\begin{aligned}
& c_{1}=\eta_{1} \eta, \quad c_{2}=c_{1}+c_{1}^{-1}, \\
& c_{3}=1-\eta_{1}^{2} \eta(2-\eta), \quad c_{4}=\eta_{1}(1-\eta), \quad c_{5}=c_{3} / c_{4} .
\end{aligned}
$$

To express $u$ as function of $\operatorname{tg} \theta(x)$, solution of the quartic equation in $u$, Eq. (2.1), is necessary. We use Eq. (2.1) from the value $x=0$ where $\theta=0$ up to a maximum value $x_{M}$ determined by $\theta=-\pi / 4$, i.e.

$$
\operatorname{tg} \theta\left(x_{M}\right)=-1 .
$$

The positive root $u_{\mathrm{M}}$ of this quartic equation gives $x_{\mathrm{M}}=\left(\ln u_{\mathrm{M}}\right) / \ln \alpha$, and tends to the value $u_{\mathrm{M}}=c_{2} /\left(1+\left(1+c_{2} / c_{5}\right)^{1 / 2}\right)$ for low temperatures. If $\phi_{2}=0$, then $u_{\mathrm{M}}=c_{2} / 2$. The value $x_{\mathrm{M}}$ corresponds to the crystal boundary point on the crystal diagonal.

\section{Shape of two-dimensional nucleus}

For the vapour saturation ratio $\alpha>1$ the step, or the crystal nucleus boundary, has a finite radius of curvature $[3,6]$. BCF have given explicit formula for the step shape from which the equilibrium crystal shape in $D=2$ dimensions can be stated. The dependence of the $y$ Cartesian coordinate on the $x$ Cartesian coordinate of the crystal nucleus boundary follows from Eq. (2.1) by integration

$$
\begin{aligned}
& y(x)=\int_{0}^{x} \operatorname{tg} \theta(x) \mathrm{d} x \\
& =\left[\ln \left(\left(c_{2}-u-u^{-1}\right)\left(u+u^{-1}+c_{5}\right)^{-1} c_{1} c_{6}^{-2} c_{7}\right)\right] / \ln \alpha,
\end{aligned}
$$


where

$$
c_{6}=1-c_{1}, \quad c_{7}=2+c_{5} .
$$

The lengths $x$ and $y$ are measured here in units of the crystal lattice constant $a_{\mathrm{L}}$, and both are scaled by $\ln \alpha$. Equation (3.1) which gives $y(0)=0$ and $y<0$ for $x>0$, is used up to the maximum value $x=x_{\mathrm{M}}$. For larger $x$ the mirror reflection in the $x=y+l / 2$ square diagonal gives the crystal nucleus shape with square symmetry [2]. Zia et al. [2,3] discussed the SOS approximation for the square lattice. The edge free energy obtained by taking the SOS for polar angle from 0 to $\pi / 4$ and using fourfold symmetry to get the rest would have a kink at $\pi / 4$, but deviation from the Ising model crystal shape is small for low temperatures and small angles [1-3]. Therefore the use of mirror reflection in square diagonal is not a bad approximation. On a square lattice it is appropriate to use as linear dimension of the nucleus its half-diameter $x_{\mathrm{L}}=x_{\mathrm{M}}-y\left(x_{\mathrm{M}}\right)=1 / 2$. At very low temperatures, if $\phi_{2}=0, x_{\mathrm{L}}$ differs little from $x_{\mathrm{M}}$ and the nucleus becomes square. If $\phi_{2}>0$, it becomes an octagon.

\section{Area of the nucleus}

The area of the crystal nucleus in $D=2$ dimensions can be expressed starting from Eq. (3.1). If the NNN interaction energy is restricted to $0<\phi_{2}<\phi_{1} / 2$, the area $a(x)$ between the $[0,1]$ or $x$ axis and the crystal nucleus boundary given by Eq. (3.1), i.e. between $x=y=0$ and $x, y(x)$, can be expressed by the integral

$$
\begin{aligned}
& a(x)=\int_{0}^{x} y(x) \mathrm{d} x=\left\{\left[\operatorname{Li}_{2}\left(c_{1} / u\right)-\mathrm{Li}_{2}\left(c_{1} u\right)+\mathrm{Li}_{2}\left(-c_{8} u\right)+\right.\right. \\
& \left.\left.-\mathrm{Li}_{2}\left(-c_{8} / u\right)\right] \ln \alpha+2 x \ln \left(c_{9}\right)\right\} / \ln \alpha
\end{aligned}
$$

where

$$
c_{8}=2 /\left[c_{5}+\left(c_{5}^{2}-4\right)^{1 / 2}\right], \quad c_{9}=\left(1+c_{8}\right) / c_{6}
$$

and

$$
\operatorname{Li}_{2}(u)=-\int_{0}^{u} \ln (1-x) \mathrm{d} x / x
$$

is the dilogarithm function [15]. For $x>0$, since $y<0$, also $a(x)<0$. At given temperature, the ratio of the actual area $n_{0}$ of the crystal nucleus to the area $l^{2}$ of the square circumscribed on it, can be expressed by

$$
n_{0} / l^{2}=1-\left[\left(y\left(x_{\mathrm{M}}\right)\right)^{2}-2 a\left(x_{\mathrm{M}}\right)\right] / x_{\mathrm{L}}^{2} .
$$

Typical values of the area ratio are given in Table for two values of the NNN interaction energy. Our computed value of $n_{0} / l^{2}$ corresponding to the BCF parameters for iodine crystal agress within $1 \%$ with the value obtained graphically by BCF. 


\section{TABLE}

Values of $x_{\mathrm{M}}$, half-diameter $x_{\mathrm{L}}$ and area ratio $n_{0} / l^{2}$ of the crystal nucleus for $\alpha=1.01$ and $\phi_{1}=0.15 \mathrm{eV}$.

\begin{tabular}{c|r|c|c|c}
\hline \hline $\begin{array}{c}\phi_{2} \\
(\mathrm{eV})\end{array}$ & $\begin{array}{c}T \\
(\mathrm{~K})\end{array}$ & $\begin{array}{c}x_{\mathrm{M}} \\
\left(a_{\mathrm{L}}\right)\end{array}$ & $\begin{array}{c}x_{\mathrm{L}} \\
\left(a_{\mathrm{L}}\right)\end{array}$ & $n_{0} / l^{2}$ \\
\hline 0.03 & 27 & $3.8873 \times 10^{3}$ & $4.5354 \times 10^{3}$ & 0.9576 \\
& 194 & $5.0668 \times 10^{2}$ & $6.2898 \times 10^{2}$ & 0.8948 \\
& 300 & $3.0537 \times 10^{2}$ & $3.9796 \times 10^{2}$ & 0.8519 \\
0.074 & 27 & $4.8377 \times 10^{3}$ & $6.4359 \times 10^{3}$ & 0.8759 \\
& 194 & $6.6287 \times 10^{2}$ & $8.9348 \times 10^{2}$ & 0.8403 \\
& 300 & $4.1393 \times 10^{2}$ & $5.6888 \times 10^{2}$ & 0.8142
\end{tabular}

\section{Activation energy}

Microscopically the diameter of the crystal nucleus is expressed in terms of a multiple of the crystal lattice constant, as in Eq. (86) of BCF [6]. Macroscopically the diameter of the critical nucleus at a given saturation ratio $\alpha$ can be measured by the distance $d$ between two opposite sign screw dislocations whose Burgers vectors protrude normal to the crystal surface, say at points $P$ and $Q$. We refer to Fig. 15 of BCF, see also Uwaha and Nozières [13]. A critical nucleus of diameter $l<d$ can pass freely between two dislocations. When $l>d$ there are two equilibrium positions: one, stable, with the nucleus boundary short piece $P A Q$, and the other, unstable, with the nucleus boundary long piece $P B Q$. The nucleus growth requires an activation energy $A_{d}$ which is half the edge free energy of the boundary piece $P B Q$ minus half the edge free energy of the boundary piece $P A Q$. The free energy of a boundary piece can be found by evaluating the area of the sector contained by the piece and the lines joining its ends to the centre of the nucleus, for the given $d / l$, as described in $\S 16$ and Appendix D of BCF. The ratio of activation energy $A_{d}$ at two dislocations, a distance $d$ apart along the $[0,1]$ crystal direction, to the activation energy $A_{0}$ for ordinary free nucleation, can be evaluated from the shape and the area functions of the step, Eqs. (3.1) and (4.1), respectively:

$$
A_{d} / A_{0}=b(x)=2\left[x\left(x_{\mathrm{L}}-y(x)\right)+2 a(x)\right] / n_{0}
$$

for $d=2\left(x_{\mathrm{L}}+y(x)\right)$ when $2 x_{\mathrm{M}}<d \leq l$, and

$$
A_{d} / A_{0}=1-b(x)
$$

for $d=2 x$ when $d \leq 2 x_{\mathrm{M}}$. As the dislocation distance $d$ increases from 0 to $2 x_{\mathrm{M}}$ the nucleus boundary coordinate $x$ increases from 0 to $x_{M}$. As $d$ increases from $2 x_{\mathrm{M}}$ to $2 x_{\mathrm{L}}=l$ the $x$ increases from $x_{\mathrm{M}}$ to $x_{\mathrm{L}}$. Corresponding to Fig. 16 of BCF where each point of the plot was obtained by using planimetry, Fig. 1 gives the calculated graphs of the ratio $A_{d} / A_{0}$ as function of $d / l$. When $d$ approaches $l$ the activation energy $A_{d}$ drops with vertical tangent to zero. At lowest temperatures the dependence of $A_{d} / A_{0}$ on $d / l$ becomes nearly polygonal. For Fig. 1 the value of the NNN interaction energy $\phi_{2}$ has been assumed near to $\phi_{1} / 2$ and hence at 

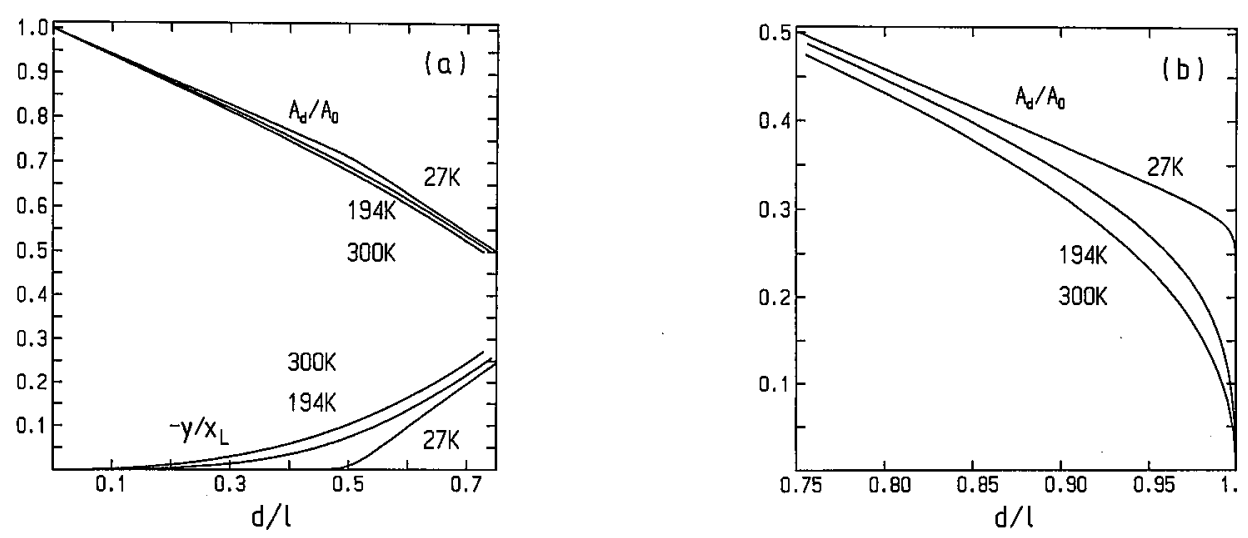

Fig. 1. Activation energy ratio $A_{d} / A_{0}$ for the growth of a step between two dislocations a distance $d$ apart (a) when $0<d \leq 2 x_{\mathrm{M}}$, (b) when $2 x_{\mathrm{M}}<d \leq l$. In the lower part of Fig. 1(a) the boundaries of the two-dimensional crystal nucleus at three temperatures are plotted (for $0<x<x_{M}$ ). The saturation ratio $\alpha=1.01$, the interaction energies are $\phi_{1}=0.15 \mathrm{eV}$ and $\phi_{2}=0.074 \mathrm{eV}$.
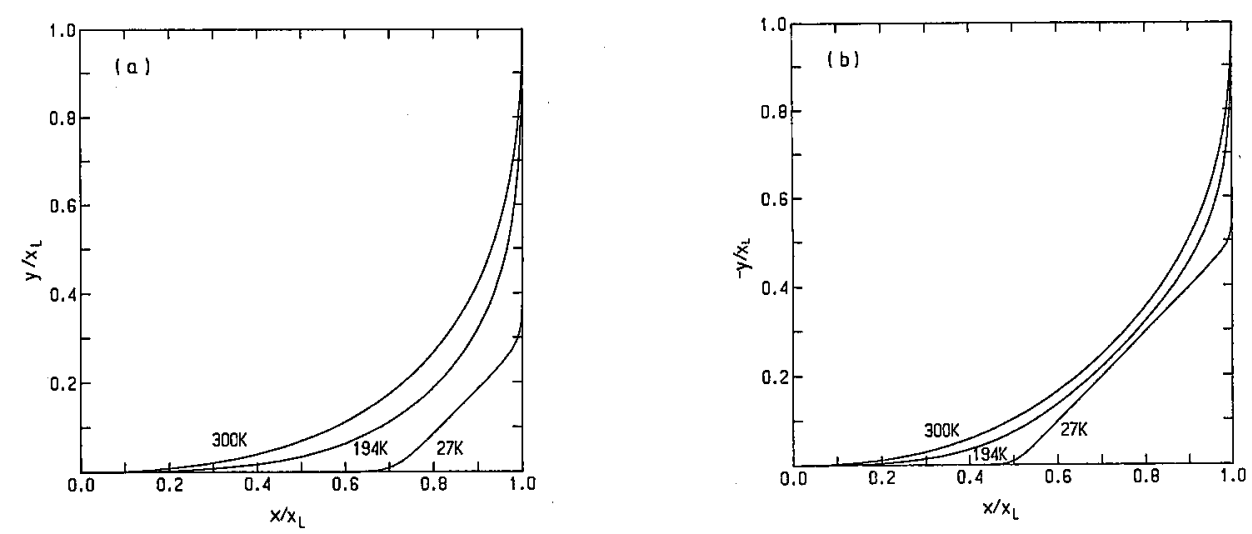

Fig. 2. One quarter of the crystal nucleus at three temperatures, for $\alpha=1.01, \phi_{1}=0.15$ $\mathrm{eV}$, and (a) $\phi_{2}=0.03 \mathrm{eV}$, (b) $\phi_{2}=0.074 \mathrm{eV}$.

low temperature the boundary of the crystal nucleus becomes almost octagonal, as seen in the lower part of Fig. 1(a), and in Fig. 2(b). At higher temperatures the curvature of the nucleus boundary becomes almost direction independent and the nucleus becomes nearly circular. For the $D=2$ Ising crystal the surface free energy has been expressed in terms of an elliptic integral by Zia and Avron [3]. 


\section{Concluding remarks}

For a rough, i.e. stepped crystal surface in unstable equilibrium with its supersaturated vapour, the $D=2$ solid-on-solid model, with NN and NNN interactions yields expression for the local mean direction of a step, and hence, by an integration, for the shape of the crystal nucleus. A second integration gives the nucleus area, and further the activation energy for nucleation between two screw dislocations.

The closed formulae for the crystal nucleus area and activation energy complement the formulae of BCF paper [6], and are useful for estimating from observed step patterns the important parameters of nucleation. The shape and activation energy of the crystal nucleus depend on the homogeneous vapour saturation ratio by simple scaling relation, while their dependence on the atomic or molecular interaction energies is more involved. The closed formulae for the nucleus shape and activation energy make possible to follow the evolution of the crystal nucleus with temperature and the activation energy dependence on screw dislocation distance. Program for computing on a square lattice the nucleus shape and activation energy can be supplied on request. Further the two-dimensional model is useful in a survey of the two-dimensional surface and cross sections of a three-dimensional crystal.

\section{References}

[1] C. Rottman, M. Wortis, Phys. Rev. B 24, 6274 (1981).

[2] J.E. Avron, H. van Beijeren, L.S. Schulman, R.K.P. Zia, J. Phys. A: Math. Gen. 15, L81 (1982).

[3] R.K.P. Zia, J.E. Avron, Phys. Rev. B 25, 2042 (1982).

[4] R.K.P. Zia, J. Stat. Phys. 45, 801 (1986).

[5] M. Holzer, Phys. Rev. B 42, 10570 (1990).

[6] W.K. Burton, N. Cabrera, F.C. Frank, Phil. Trans. Roy. Soc. London, Ser. A 243, 299 (1951).

[7] C. Jayaprakash, W.F. Saam, S. Teitel, Phys. Rev. Lett. 50, 2017 (1983).

[8] C. Rottman, M. Wortis, J.C. Heyraud, J.J. Métois, Phys. Rev. Lett. 52, 1009 (1984).

[9] C. Rottman, M. Wortis, Plys. Rev. B 29, 328 (1984).

[10] C. Rottman, M. Wortis, Phys. Rep. 103, 59 (1984).

[11] C. Jayaprakash, C. Rottman, W.Z. Saam, Phys. Rev. B 30, 6549 (1984).

[12] H.J. Schulz, J. de Physique 46, 257 (1985).

[13] M. Uwaha, P. Nozières, J. de Physique 48, 407 (1987).

[14] P. Bennema, J. Crystal Growth 69, 182 (1984).

[15] A. Devoto, D.W. Duke, Rivista del Nuovo Cim. 7 (6), 1 (1984). 